\title{
Prescripción de Medicamentos en Pacientes Atendidos en Instituciones de Mediano y Alto Nivel de Complejidad
}

\section{Drug prescription patterns in patients attended at a middle- and high-complexity level institution}

Jefferson A. Buendía- Rodriguez ${ }^{1}$, José J. Lopez-Gutierrez², Oscar A. Garcia-Vega ${ }^{3}$, Jorge Diaz-Rojas² y Juana P. Sánchez-Villamil ${ }^{4}$

1 Facultad de Medicina. Universidad Antonio Nariño.

2 Departamento de Farmacia, Facultad de Ciencias. Universidad Nacional de Colombia. Sede Bogotá.

3 Departamento de Ciencias Fisiológicas. Facultad de Medicina. Universidad Nacional de Colombia

5 Hospital Rafael Uribe Uribe. Bogotá, Colombia.

Recibido 19 Diciembre 2007/Enviado para Modificación 19 de Julio 2008/Aceptado 3 Septiembre 2008

\section{RESUMEN}

Objetivo Determinar los patrones de prescripción de medicamentos ambulatorios autorizados en pacientes afiliados a una EPS y atendidos por IPS de mediano y alto nivel de complejidad.

Métodos Se realizo un estudio analítico transversal donde se evaluaron las prescripciones de medicamentos en 331 IPS de segundo y tercer nivel de complejidad en 27 departamentos durante el 2006.

Resultados Se analizaron 38863 prescripciones de medicamentos de 3663 pacientes. El 61,4\% de estas provienen de IPS de tercer nivel de complejidad y principalmente de pacientes afiliados al régimen contributivo. El promedio de medicamentos por formula medica fue de 2,2 (IC $95 \%$ : 2,1 a 2,2 \%), la proporción de antibióticos por fórmula médica del 29,2 \% (IC $95 \%$ : 28,7-29,6), de medicamentos POS del 64,2 \% (IC 95 \% 63,7-64,6 \%) y de inyectables del 22,1 \% (IC 95 \% 21,725,5 \%). Más de la mitad de los medicamentos, 62,1 \% (IC 95 \% 61,5-62,7 \%), corresponden a agentes anti-infecciosos, antineoplasicos y del tracto alimentario .El consumo de medicamentos fue de 8,39 Dosis Diarias Definidas (DDD) /1 000 usuarios/día y el costo por DDD fue de \$ 5216

Conclusión Este análisis permitió identificar medicamentos cuya frecuencia de formulación no se correlaciono con el perfil epidemiológico como agentes inmunomoduladores y hormona de crecimiento. Existen diferencias en la frecuencia y cantidad de DDD de medicamentos autorizados por régimen de afiliación lo cual pude ser evidencia de barreras de acceso a los medicamentos en la población.

Palabras Clave: Prescripción de medicamentos, utilización de medicamentos (fuente: DeCS, BIREME). 


\section{ABSTRACT}

Objective Determining prescription patterns for outpatient medication authorised for patients affiliated to an EPS and assisted by medium- and high-level complexity IPS.

Methods This was a cross-sectional study where medication prescription was evaluated in 331 second- and third-level complexity hospitals from 27 Colombian departments during 2006.

Results 38863 prescriptions for 3663 patients' medication were analysed. 61,4\% came from third-level complexity hospitals, mainly for patients affiliated to contributionbased regimes. Average prescribed medication per person was 2,2 (2,1-2,2 $95 \%$ $\mathrm{Cl})$, the percentage of antibiotics formulated by prescription was 29,2 \% (28,7-29,6 $95 \% \mathrm{Cl})$, essential prescribed medicines accounted for $64,2 \%(63,7-64,695 \% \mathrm{Cl})$ and injectable medicines was $22,1 \%(21,7-25,5 \%)$. More than half the medications $(62,1 \% ; 61,5-62,795 \% \mathrm{Cl})$ were in ATC groups such as anti-infectious agents, immunomodulating agents and medications for the alimentary and metabolic tract. DU90\% consisted of 64 medications, medication consumption being 8,39 daily de-fined doses (DDD)/1 000 patients, costing 5216 Colombian pesos per DDD.

Conclusions This analysis led to identifying medications whose formulation frequency did not correlate with an epidemiologic profile as immunomudulator and growthhormone (somatotropin) agents. There were differences in the frequency and quantity of DDD medications authorised by type of affiliation which could thus be providing evidence of obstacles to the population having access to drugs/medicaments.

Key Words: Drug use, prescription, drug (source; MeSH, NLM).

A pesar del constante desarrollo que han tenido los medicamentos en los últimos 50 años y de su efecto positivo en la disminución de la morbimortalidad, existe también suficiente evidencia acerca del riesgo que genera su uso inadecuado (1). Este problema ha alcanzado una gran magnitud; un documento de la OMS señala que en el mundo, más del 50\% de todos los medicamentos, se prescriben, dispensan, venden o consumen de manera inadecuada (2).Existen evidencia que estos problemas, derivados de patrones de prescripción inadecuados, se asocian a reducción en los beneficios esperados de los medicamentos y un aumento en los costos de atención en salud (2) Sin embargo, para poder abordar este problema es necesario contar con información suficiente, valida y confiable respecto a los patrones de prescripción de medicamentos y los factores que inciden en los mismos. Esta información es limitada en nuestro país dado que la mayoría de aproximaciones realizadas se han generado a partir de estudios de cualitativos, de baja calidad y en población seleccionadas (3). 
Este trabajo pretende describir los patrones de prescripción de medicamentos - en términos de consumo e indicadores OMS de prescripción - en población ambulatoria , afiliados a una entidad prestadora de servicios de salud , y atendida en Instituciones Prestadoras de Servicios de Salud (IPS) de mediano y alto nivel de complejidad durante el 2006. Así mismo se busca realizar un análisis exploratorio de posibles variables sociodemográficas asocia-das a la prescripción de medicamentos en esta población.

\section{METODOLOGÍA}

Se realizo un estudio analítico de corte transversal; utilizando como fuente de información todos los registros de medicamentos autorizados en pacientes afiliados a una Entidad Promotora de Salud (EPS) durante el 2006 y atendidos por 331 IPS de segundo y tercer nivel de complejidad en 27 departamentos. Esta EPS contaba con aproximadamente 900000 afiliados para el 2006, el 57 $\%$ de ellos pertenecientes al régimen contributivo y el resto al subsidiado; y realizaba sus actividades en 29 Departamentos del país.

De cada registro de autorización de medicamento se contaba con información de edad y sexo del paciente, régimen de afiliación, departamento, nombre común internacional del medicamento prescrito, cantidad de medica-mento prescrito, valor unitario del medicamento, forma farmacéutica, con-centración del medicamento y diagnostico principal del paciente. Esta base de datos fue sometida a dos fases secuenciales de depuración por revisores independientes utilizando una matriz de validación, en las que se evaluó: la consistencia de cada campo (presencia de datos dentro de los rangos establecidos de respuesta para cada campo) y coherencia (relación lógica existente entre dos campos correlacionados por ejemplo diagnóstico con sexo) validando esta información frente a los registros originales en papel. Una vez depurada la base de datos, los diagnósticos principales relacionados se categorizaron utilizando la clasificación internacional de enfermedades (CIE-10) y los medicamentos según la clasificación anatómica, terapéutica y quí-mica (ATC) de la OMS (4).

Análisis Estadístico: Para describir las variables continuas se utilizaron medias y medianas como medidas de tendencia central, y desviación estándar, rangos o intervalos de confianza a un nivel del 95 \% como medidas de dispersión. Las variables discretas, nominales y ordinales se describieron con razones y proporciones, calculando sus intervalos de confianza a un nivel del $95 \%$ para describir su dispersión. 
Para determinar el consumo de medicamentos se utilizo como medida la Dosis Diarias Definidas (DDD) por medicamento, valor el cual se calculo según la metodología recomendada por la OMS (4). Para evaluar la calidad de la prescripción de medicamentos se calculo la frecuencia acumulada de medicamentos (DU90) según la metodología definida por Bergman (5). Con el fin de determinar variables sociodemográficas asociadas a la prescripción de medicamentos se utilizo la técnica de componentes principales siguiendo las recomendaciones de Perez-Lopez (6).Los análisis estadísticos fueron desarrollados utilizando Intercooled STATA 8.0 (7)

\section{RESULTADOS}

Se analizaron 38861 prescripciones de medicamentos generadas por un total de 3663 pacientes durante el 2006, cuyo promedio de edad fue 41,5 años (41,3-41,7 IC 95 \%); las características sociodemográficas y lugar de atención de esta población se presentan en la Tabla 1. Alrededor del 80,3 \% de los diagnósticos principales asociados a los medicamentos autorizados se agruparon en las categorías de enfermedades neoplásicas (39,9 \%), infecciosas (13,9 $\%)$, enfermedades neurológicas (10,5\%), enfermedades cardiovasculares (10,5 \%) y enfermedades endocrinas (5,6 \%); la distribución de estos grupos de enfermedades por sexo y régimen de afiliación se presenta en la Tabla 2.

Tabla 1. Características sociodemográficas de la población

\begin{tabular}{lccc}
\hline \multicolumn{1}{c}{ Variable } & $\mathrm{n}$ & $\%$ & IC $95 \%$ \\
\hline Sexo Femenino & 1985 & 54,2 & 59,9 a 57,1 \\
Ariliados al régimen Contributivo & 2004 & 54,7 & 55,3 a 54,1 \\
Lugar de atención & & & \\
$\quad$ IPS de segundo nivel de complejidad & 1410 & 61,4 & $59,8-62,9$ \\
IPS de tercer nivel de complejidad & 2249 & 38,5 & $36,9-40,0$ \\
Lugar de Residencia & & & \\
Bogotá D,C & 1524 & 42,0 & $39,9-43,2$ \\
Bolivar & 323 & 8,9 & $7,8-9,7$ \\
Huila & 239 & 6,6 & $5,7-7,3$ \\
Atlántico & 173 & 4,7 & $4,0-5,4$ \\
Tolima & 139 & 3,8 & $3,1-4,4$ \\
Otros & 1265 & 33,7 & $32,9-36,0$ \\
\hline
\end{tabular}

Se registraron un total de 38861 prescripciones autorizados durante el 2006, que corresponden a 919 presentaciones de medicamentos o 517 fármacos o principios activos. Se encontró un promedio de medicamentos por formula medica de 2,23 (IC $95 \%$ : 2,1 a 2,2 medicamentos por formula médica). El porcentaje de inyectables fue de 22,18 \% (IC $95 \%$ : 21,7-25,5\%), el de antibióticos formulados por prescripción fue del 29,2 \% (IC 95 \%: 28,7-29,6 
\%) y el de medicamentos esenciales (POS) por prescripción fue del 64.2 \% (IC 95\% 63,7-64,7 \%). Respecto al comportamiento temporal de las pres-cripciones de medicamentos durante el año, en ambos regimenes, se caracterizo por un aumento progresivo en las autorizaciones a medida que transcurre en año; con un pico en el mes de Noviembre; y descendiendo en diciembre, descenso mas marcado en el régimen subsidiado.

Tabla 2, Distribución de los principales grupos de enfermedades asociadas con la prescripción de medicamentos según sexo y régimen de afiliación

\begin{tabular}{lcccccccc}
\hline \multirow{2}{*}{ Categoria } & \multicolumn{4}{c}{ Contributivo } & \multicolumn{3}{c}{ Subsidiado } \\
\cline { 2 - 8 } & \multicolumn{2}{c}{ Femenino } & Masculino & Femenino & Masculino \\
\hline & $\mathrm{N}$ & $\%$ & $\mathrm{n}$ & $\%$ & $\mathrm{n}$ & $\%$ & $\mathrm{n}$ & $\%$ \\
\hline E, neoplásicas & 701 & 37,2 & 302 & 24,0 & 958 & 62,3 & 329 & 31,1 \\
E, infecciosas & 107 & 5,6 & 161 & 12,8 & 194 & 12,6 & 334 & 31,6 \\
E, cardiovasculares & 251 & 13,3 & 235 & 18,7 & 59 & 3,8 & 59 & 5,5 \\
E, neurológicas & 210 & 11,1 & 191 & 15,2 & 91 & 5,9 & 96 & 9,0 \\
E, endocrinas & 184 & 9,7 & 68 & 5,4 & 33 & 2,1 & 36 & 3,4 \\
\hline
\end{tabular}

Midiendo el consumo de medicamentos según el número de Dosis Diarias Definidas (DDD) los principales medicamentos prescritos (74,9\% del total) se agruparon, utilizando las categorías de la clasificación anatómica, terapéutica y química (ATC), en los grupos de medicamentos antineoplasicos e Inmunomoduladores, medicamentos del tracto alimentario y medicamentos antiinfecciosos como se observa en la Tabla 3. Mientras los grupos de medicamentos con mayor costo asociado fueron en su orden los medicamentos antiinfecciosos, antineoplásicos y medicamentos del sistema nervioso.

Los medicamentos que dan cuenta del $90 \%$ del total de Dosis Diarias Definidas en la población (DU90 \%) correspondieron a 64 Medicamentos de los cuales el 53,1 \% no pertenecen al plan obligatorio de salud (POS) o listado de medicamentos esenciales (IC95 \%: 45,5 a 63,8\%). El DU90 \% estuvo conformado por 38 medicamentos para el régimen subsidiado y 64 para el contributivo, 59 medicamentos para el sexo masculino y 62 para el femenino. El promedio nacional de consumo de medicamentos fue de 8.3 Dosis Diarias Definidas/1000 usuarios/día siendo los departamentos de Cauca, Santander, Nariño, Sucre, Quindío, Risaralda, Atlántico, Valle y Meta quienes presentaron promedios de consumo superiores al promedio nacional.

El costo global por DDD en general fue de \$ 5216 pesos colombianos. El costo por DDD se incrementó a medida que envejece la población aumentando alrededor de $\$ 200$ pesos colombianos por cada año de vida; esto a expensas principalmente por la alta frecuencia y costo de los medicamentos 
cardiovasculares y hormonales. Igualmente se presenta un pico en el costo/ DDD entre los 16 y 30 años debido a la cantidad de medicamentos antiretrovirales en este grupo etareo. Los medicamentos con mayores costos por DDD, dentro de los medicamentos listados en el DU90 \%, se presentan en la Tabla 4.

El análisis de componentes principales permitió explorar asociaciones entre variables sociodemográficas con la prescripción de medicamentos según grupos ATC. Se puedo evidenciar dos agrupaciones la primera conformada por el sexo femenino, régimen contributivo, edades entre 40 a 75 años y prescripción de antineoplásicos, medicamentos hormonales, cardiovasculares y músculos esqueléticos, agrupación esperada por las consecuencias del aumento de patología neoplásica y cardiovascular en la postmenopausia; y la segunda por el sexo masculino, régimen subsidiado y medicamentos antiin-fecciosos.

Tabla 3. Distribución cantidad de dosis diarias definidas de los grupos de medicamentos según clasificación anatómica, terapéutica y química (ATC)

\begin{tabular}{|c|c|c|c|c|c|}
\hline $\begin{array}{c}\text { Grupos de medicamentos } \\
\text { según ATC }\end{array}$ & $\begin{array}{r}\text { Dosis } \\
\text { Diarias } \\
\text { Definidas } \\
\text { (DDD) }\end{array}$ & $\%$ & $\stackrel{\%}{\text { Acumulado }}$ & $\begin{array}{r}\text { DDDV } \\
100000 \\
\text { usuarios/ } \\
\text { dia }\end{array}$ & $\begin{array}{l}\% \text { del } \\
\text { costo } \\
\text { total }\end{array}$ \\
\hline $\begin{array}{l}\text { Antinecplásicos e } \\
\text { inmunomoduladores }\end{array}$ & 849585 & 52,8 & 52,9 & 195,7 & 36,6 \\
\hline Tracto alimentario y metabolismo & 194821 & 12,1 & 65,0 & 44,8 & 2,4 \\
\hline Antiinfecciosos de uso sistémico & 160030 & 9,9 & 74,9 & 36,8 & 42,9 \\
\hline Sistema nervioso & 96635 & 6,0 & 81,0 & 22,2 & 5,5 \\
\hline Sistema cardiovascular & 84817 & 5,2 & 86,2 & 19,5 & 1,1 \\
\hline $\begin{array}{l}\text { Sistema genitourinario/hormonas } \\
\text { sexuales }\end{array}$ & 66304 & 4,1 & 90,3 & 15,2 & 0,7 \\
\hline $\begin{array}{l}\text { Hormonales sistémicos, excepto } \\
\text { insulinas y hormonas sexuales }\end{array}$ & 63180 & 3,9 & 94,3 & 14,5 & 4,5 \\
\hline $\begin{array}{l}\text { Sangre y órganos } \\
\text { hematopoyéticos }\end{array}$ & 47919 & 2,9 & 97,2 & 11,0 & 1,5 \\
\hline Sistema músculo esquelético & 18800 & 1,1 & 98,4 & 4,3 & 2,3 \\
\hline Sistema respiratorio & 8455 & 0,5 & 98,9 & 1,9 & 0,8 \\
\hline Organos de los sentidos & 8240 & 0,5 & 99.4 & 1,9 & 0.3 \\
\hline Medicamentos Varios & 3697 & 0,2 & 99,7 & 0,8 & 0,6 \\
\hline $\begin{array}{l}\text { Antiparasitarios, insecticidas y } \\
\text { repelentes }\end{array}$ & 2835 & 0,1 & 99,9 & 0,6 & 0,2 \\
\hline Medicamentos dematológicos & 1640 & 0,1 & 100,0 & 0,3 & 0.3 \\
\hline Total general & 1606958 & 100,0 & & & 100,0 \\
\hline
\end{tabular}


Tabla 4. Medicamentos pertenecientes al DU90\% cuyo costo por dosis diaria definida fue superior al percentil 95 de la distribución de costos por dosis diaria definida

\begin{tabular}{lrrrr}
\hline \multicolumn{1}{c}{ Medicamento } & $\begin{array}{c}\text { Cantidad de } \\
\text { Dosis Diarias } \\
\text { Definidas } \\
\text { (DDD) }\end{array}$ & $\begin{array}{c}\text { \% } \\
\text { DDD }\end{array}$ & $\begin{array}{c}\text { Costo Total en } \\
\text { pesos } \\
\text { Colombianos } \\
\text { (\$) }\end{array}$ & $\begin{array}{c}\text { Costo } \\
\text { DD } \\
\text { (\$) }\end{array}$ \\
\hline Infliximab & 3200 & 0,2 & 222602343 & 69563 \\
Acido Micofenolico & 3240 & 0.2 & 152195820 & 46974 \\
Hormona de Crecimiento & 14966 & 0,9 & 652672849 & 43610 \\
Atazanavir & 3915 & 0.2 & 128433309 & 32805 \\
Otros & 1423652 & 88.4 & 4053953891 & 6438 \\
\hline Total & 1448973 & 90,0 & 5209858212 & \\
\hline
\end{tabular}

\section{DISCUSIÓN}

En el presente estudio se evidencio que mas de la mitad de los fármacos (62,1 \%, IC 95 \%: 61,5- 62,7 \%) prescritos en la población estudiada se agruparon en las categorías ATC correspondientes a agentes antiinfecciosos, agentes antineoplásicos e inmunomoduladores y medicamentos del tracto alimentario y metabólico. Esto refleja claramente el impacto que tienen las patologías crónicas, especialmente las de alto costo tanto en las Entidades Promotoras de salud; entre las cuales medicamentos como los antineoplásicos y antinfecciosos dieron cuenta del $36 \%$ de la formulación pero el 76 \% del costo. Esta falta de simetría entre la frecuencia y el costo, dado por el alto costo por medicamentodía de estos grupos de medicamentos, revela la necesidad de fortalecer las actividades de promoción y prevención como estrategias resolutivas a largo plazo para evitar el desborde del gasto medico en el sistema general de seguridad social de salud (SGSSS) (8). Así mismo, coloca de manifiesto la necesidad de evaluar los costos de medicamentos para patologías de alto costoespecialmente antineoplásicos- que dada su tendencia creciente de consumo (8-9), deben tener costos más bajos para que el precio de estos medicamentos no se constituya en una barrera de acceso a los medicamentos y afecten las metas a largo plazo del país respecto al control del patologías transmisibles y crónicas no transmisibles.

El promedio de medicamentos prescritos por formula medica de 2,2 fue similar al encontrado de 2,0 por el estudio UN-INVIMA (9) y al reportado en el estudio de monitoreo de la situación farmacéutica en Colombia (MSF)(10) que fue de 2,3. Nosotros encontramos una proporción de inyectables (22,1 \%), mucho mayor que la encontrada en el estudio UN-INVIMA (9,5 \% de las prescripciones) y en el estudio de MSF (13,3\%). El porcentaje de anti-bióticos formulados también fue mayor que lo encontrado por el estudio UN- 
Minproteccion (25,3\%) y el recomendado por OMS (25\%) pero menor que el encontrado por el estudio MSF (30 \%). El porcentaje de prescripción de medicamentos esenciales (64,2 \%) fue menor que el encontrado por el estudio UNMinProteccion y que del estudio MSF (94,2 \%). Estas diferencias son debidas a variaciones en las fuentes de información utilizadas en cada proyecto; mientras en nuestro estudio fueron registros de IPS principalmente de tercer nivel de complejidad en los estudios UN-MinProteccion y MSF acudieron a copias de prescripciones de médicos principalmente generales y registros de farmacias de IPS de primer nivel de complejidad; donde dada la atención de patologías de menor severidad, la utilización de medicamentos inyectables, antibióticos y medicamentos no POS es menos fre-cuente que en centros de mayor nivel de complejidad.

El DU90 \% fue de 64 medicamentos, resultado similar en cantidad al encontrado por el estudio UN-Minproteccion de 67, pero diferente en los medicamentos que lo conforman dada las diferencias en las fuentes de información comentadas anteriormente. Este análisis del DU90 \% permitió identificar medicamentos (hormona de crecimiento, interferones e inmunoestimulantes) cuya frecuencia inesperada no se correlaciono con el perfil de morbilidad de la población.

Los resultados del análisis multivariado demuestran que el sexo determino patrones de prescripción en la población tan característicos como el observado en mujeres postmenopausicas (principalmente del régimen contributivo formuladas con medicamentos cardiovasculares y antineoplasicos) y en hombres adultos jóvenes (principalmente del régimen subsidiado formulados con antiinfecciosos). Esto demuestra que esta variable más que la edad es una determinante en los patrones de prescripción de medicamentos y que por ende las intervenciones orientadas al uso racional de medicamentos deben reconocer las diferencias de género en el diseño de políticas tendientes a mejorar el uso de medicamentos en la población.

Respecto al régimen de afiliación se observo que existen diferencias en el numero de medicamentos que dan cuenta del $90 \%$ del las dosis diarias definidas (DU90 \%); el cual estuvo constituido por 38 medicamentos en subsidiado y 64 en contributivo; brecha que se mantiene aun cuando esta misma lista se hace ordenando los medicamentos por su costo total (42 subsidiado y 81 contributivo). Precisamente son diferencias en el acceso a medicamentos las que pueden explicar porque en el régimen contributivo el $90 \%$ de las dosis diarias definidas (DU90 \%); son generadas por 64 medicamentos mientras en el subsidiado este valor solo es de 38. Estas diferencias han sido notadas también 
por estudios previos. Por ejemplo, un estudio de la defensoría del pueblo reporta que solo el 46,6 \% de los medicamentos en el régimen subsidiado eran entregados completamente mientras que en el contributivo ese valor era del 55 \% (11-12). Estas diferencias contrastan con el espíritu de listado de medicamentos esenciales o POS el cual es único para ambos regímenes de afiliación (12); colocando de manifiesto que la restricción realizada en la legislación respecto a las actividades y no en los medicamentos incluidas en el plan obligatorio de salud para el régimen subsidiado pueden estar impactando negativamente los patrones de prescripción de medicamentos y el posible beneficio que puede obtener estas tecnologías en la población.

Dentro de las limitaciones de este estudio esta el posible sesgo de clasificación (o información) dado que se recurrió a registros secundarios como las autorizaciones de medicamentos y no se reviso la historia clínica original; siendo posible que se registre como diagnostico principal una patología que aqueja al paciente pero que no es la razón de prescripción del medicamento registrado. (Vg. Se puede haber registrado infección por VIH sin especificación y el medicamento registrado es un antimicótico prescrito para manejar una Micosis secundaria). Así mismo, tal como ha sido evidenciado con otros registros de atención medica pueden existir problemas de registro por falta de educación adecuada al medico tratante en el manejo de la codificación CIE-10 que se traduce en errores sistemáticos en la asignación de códigos diagnósticos en la historia clínica. Estos errores generan sesgos de clasificación que pueden sobrestimar indicadores de morbilidad calculados; por lo cual y dado que no era un objetivo del estudio no se realizo análisis de indicación-prescripción o prescripción-indicación de medicamentos. También es posible que haya ocurrido subregistro ocasionado por el no envió de registros, lo cual no fue cuantificado ni estimado el efecto sobre los patrones de prescripción encontrados •

\section{REFERENCIAS}

1. Organización Mundial de la Salud. Perspectivas políticas sobre medicamentos de la OMS. Promoción del uso racional de medicamentos: componentes centrales. Geneva: Organización Mundial de la Salud; 2002.

2. Organización Panamericana de la Salud. Estrategia Revisada de la OMS en Materia de Medicamentos. Washington: OPS; 1998.

3. Orozco J, Holguin E, Cardenas ML, García O. Estudio de Evaluación de intervenciones para modificar hábitos de prescripción 1ra Edición, OPS. Bogota; 2004.

4. WHO Collaborating Centre for Drug Statistics Methodology [Internet]. Disponible en: http:// www.whocc.no/atcddd/. Consultado Agosto del 2008

5. Bergman U, Popa C, Tomson $Y$, Wettermark B, Einarson TR, Aberg H, Sjöqvist F. Drug utilization $90 \%$ - a simple method for assessing the quality of drug prescribing. Eur $\mathrm{J}$ Clin Pharmacol 1998; 54 (2): 113-9. 
614 REVISTADE SALUD PÚBLICA · Volumen 10 (4), Septiembre 2008

6. Perez Lopez C. Tecnicas de Analisis Multivariante de datos. Madrid: Pearson Prectice Hall Eds. Vol.1; 2004. p. 456-672.

7. STATA 8.0. STATA Corporation, Inc. College Station, Texas, USA; 2003.

8. Whitehead M.The concepts and principles of equity in health. International Journal of Health Services 1992; 22(3):429-445

9. Orozco J, Holguin E, Cardenas ML, García O. Informe final Estudio de Utilizacion de Medi-camentos. Convenio de Cooperación Interadministrativo 172/03. Ministerio de la Proteccion Social. Universidad Nacional de Colombia. Ministerio de la Proteccion Social, Bogota. Vol 1; 2004. p 12-56.

10. Ministerio de la protección social de Colombia-OPS. Monitoreo de la situación farmacéutica en Colombia. 2003. Ministerio de la Proteccion Social, Bogota; 2003. pp. 6-10.

11. Mejía S, Vélez AL, Arango MC, Buriticá O. La Política Farmacéutica Nacional en Colombia y la Reforma de la Seguridad Social: Acceso y Uso Racional de Medicamentos. Cuadernos de Saúde Pública 2002; 18 (4): 1025-29

12. Mejía-Mejía, Aurelio, Sánchez-Gandur, Andrés F, Tamayo-Ramírez JC. Equidad en el Acceso a Servicios de Salud en Antioquia, Colombia. Rev. salud pública (Bogotá) 2007; 9 (1): 26-38. 